\title{
Zuber's Person-Centered Care Clinical Governance Standards for Hospitals
}

\author{
Zuber Mujeeb Shaikh \\ Director, Corporate Quality Improvement, Patient Safety and Risk Management \\ Dr. Sulaiman Al-Habib Medical Group, \\ Riyadh-11643, Kingdom of Saudi Arabia \\ ORCID-ID- https://orcid.org/0000-0002-1491-8379 \\ Email: drzuber5@yahoo.co.in
}

Area/Section: Health Sciences.

Type of the Paper: Conceptual Paper.

Type of Review: Peer Reviewed as per $|\mathrm{C}| \mathrm{O}|\mathrm{P}| \mathrm{E} \mid$ guidance.

Indexed in: OpenAIRE.

DOI: https://doi.org/10.5281/zenodo.4772988.

Google Scholar Citation: IJHSP

\section{How to Cite this Paper:}

Shaikh, Zuber Mujeeb, (2021). Zuber's Person-Centered Care Clinical Governance Standards for Hospitals. International Journal of Health Sciences and Pharmacy (IJHSP), 5(1), 117129. DOI: http://doi.org/10.5281/zenodo.4772988.

International Journal of Health Sciences and Pharmacy (IJHSP)

A Refereed International Journal of Srinivas University, India.

Crossref DOI : $\underline{\text { https://doi.org/10.47992/IJHSP.2581.6411.0069 }}$

(C) With Author.

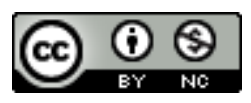

This work is licensed under a Creative Commons Attribution-Non-Commercial 4.0 International License subject to proper citation to the publication source of the work.

Disclaimer: The scholarly papers as reviewed and published by the Srinivas Publications (S.P.), India are the views and opinions of their respective authors and are not the views or opinions of the SP. The SP disclaims of any harm or loss caused due to the published content to any party. 


\title{
Zuber's Person Centered Care Clinical Governance Standards for Hospitals
}

\author{
Zuber Mujeeb Shaikh \\ Director, Corporate Quality Improvement, Patient Safety and Risk Management \\ Dr. Sulaiman Al-Habib Medical Group, \\ Riyadh-11643, Kingdom of Saudi Arabia \\ ORCID-ID- https://orcid.org/0000-0002-1491-8379 \\ Email: drzuber5@yahoo.co.in
}

\begin{abstract}
Purpose: To establish the Clinical Governance standards for hospitals based on the Person-Centered Care concepts since it is crucial in improving standards of care that patients receive.

Objective: To develop Person Centered Care Clinical Governance Standards for Hospitals.

Method: This is a review of literature study and the data were collected with comprehensive searches in the online databases of Google Scholars and Research Gate.

Conclusions: The study concluded with "Zuber's Person-Centered Care Clinical Governance Standards for Hospitals."

Keywords: Person Centered Care, Clinical Governance, Standards, Hospital, Zuber

\section{INTRODUCTION :}

Clinical Governance is a system through which organizations are accountable for continuously improving the quality of their services and safeguarding high standards of care creating an environment in which in clinical care will thrive.

In healthcare service industry, at the moment there are several national and international standards for hospitals, laboratories, blood banks, and for some clinical specialties but unfortunately, the standards for Person Centered Care Clinical Governance are still not available for hospitals in order to maintain quality, patient safety, risk management and patient experience.

Clinical Governance is an umbrella term which encompasses a range of activities in which clinicians should become involved in order to maintain and improve the quality of the care they provide to patients and to ensure full accountability of the system to patients. Clinical Governance addresses those structures, systems and processes that assure the quality, accountability and proper management of an organization's operation and delivery of service.
\end{abstract}

\section{REVIEW OF LITERATURE :}

Patients, along with their relatives where appropriate, should be equal partners in planning, developing, and assessing care to ensure that it is the most appropriate for their needs. A safe experience may not be a personcentred one and a good experience might not be a safe one. Person-centred care also includes complaints and a process whereby these are reviewed and responded to in a timely fashion. Surveys of patients can form an important part of person-centred care, although these can be more difficult in the field of anesthesia, where many other factors influence the experience of a patient undergoing surgery.

The concept of patient satisfaction is since the 1960s, but there was no research conducted actively until 1970s and however the research scholars initiated active research in the early 1980s and replaced the idea of "quantity of life" with a more patient-centered concept of quality of life (Ekram S, Rahman, 2006) [1].

Researchers had revealed that accreditation showed positive impact on hospital emergency transport services (Shaikh, 2016) [2], Department Services (Shaikh, 2017) [3], Dietary Services (Shaikh, 2017) [4], Laboratory Department Services (Shaikh, 2017) [5], Emergency Department Services (Shaikh, 2017) [6], In-Patient Department Services (Shaikh, 2017) [7], Hemodialysis Department Services (Shaikh, 2017) [8], Radiology Department Services (Shaikh, 2017) [9], Pharmacy Department Services (Shaikh, 2017) [10], Out-Patient Department (Shaikh, 2017) [11]. 
Researchers had conducted several studies earlier to understand the impact of hospital accreditation on the patients' experience. It has been proved that the accreditation has a positive impact on the patients' experience of hospital Emergency Transport Services (Shaikh, 2018) [12], Hemodialysis services (Shaikh, 2019) [13] and Emergency Department Services, (Shaikh, 2019) [14].

The accreditation of hospitals revealed improvement in the Human Resources Department Services (Shaikh, 2017) [15], Occurrence Variance Report or Incident Reports system (Shaikh, 2018) [16] and Laboratory \& Blood Bank performance (Shaikh, 2018) [17], Critical Care Unit Outcome Quality Measures, (Shaikh, AlOmari, A., \& Ahmed, 2018) [18].

The Planetree Person Centered Care accreditation of an accredited (national and international) hospital revealed a positive impact on its services (Shaikh, Al-Omari, \& Ahmed, 2018) [19], and Person-Centered Care Design has a positive impact on the healing process [20].

The researchers have revealed that the accreditation has improved patient satisfaction, experience and outcomes [21, 22, 23, 24, 25, 26, 27, 28, 29, 30, 31, 32, 33, 34, 35, 36, 37, 38, 39, 40, 41, 42, 43, 44, 45, 46, $47,48,49]$.

The researchers have revealed that clinical governance framework contribute to the changing role of nurse leaders [50]. Evidence indicates consumer partnerships lead to improved service design, quality and innovation [51].

Since the seminal 1978 Declaration of Alma Ata [52], it has become widely accepted that citizens have both rights and duties to participate in planning and implementing their health care and there has been a shift towards recognizing clinicians, managers, consumers and carers as partners in the health system [53].

One of the research studies showed that there exist a variety of challenges for the implementation of clinical governance and to successfully implement a health policy, its infrastructure needs to be created [54].

Clinical governance encompasses the domains of clinical effectiveness, risk management, patient safety and consumer engagement, and should address the priority areas and accreditation requirements of any national regulatory bodies [55]. It requires good multidisciplinary working and a willingness to reflect on and learn from errors to achieve a patient-centred and safer system [56].

Health care should move toward quality improvement and safe practice through the effective integration of CRM in organizational process [57]. The process of audit is part of clinical governance, by which the healthcare organizations continuously improve the quality of their services and safeguarding high standards by creating an environment in which excellence in clinical care will flourish [58]. Clinical Effectiveness has made easy the Clinical Governance [59]. Clinical governance that could be utilized by multidisciplinary teams to develop and maintain an organizational culture of quality [60].

\section{ZUBER'S PERSON CENTERED CARE CLINICAL GOVERNANCE STANDARDS FOR HOSPITALS:}

These standards are divided in to 11 Chapters and 97 Standards as follows:

1. Governance and Leadership- (18 Standards)

2. Partnerships with Consumer- (18 Standards)

3. Personnel- (11 Standards)

4. Clinical Risk Management- (9 Standards)

5. Clinical Practice- (17 Standards)

6. Education and Training- (4 Standards)

7. Clinical Audit- (5 Standards)

8. Clinical Effectiveness- (4 Standards)

9. Research and Development- (3 Standards)

10. Openness- (5 Standards) 


\section{Information Management- (3 Standards)}

\section{GOVERNANCE AND LEADERSHIP :}

1.1 The Board of Governance of the organization should provide the leaders of the hospital all resources to develop and implement a culture of quality improvement, patient safety, risk management, patient experience and a Blame Free Culture.

1.2 The Board of Governance should ensure that the hospital leaders have involved their patients, families, carers, staff, stakeholder and community leaders, in planning, developing and revising the Mission, Vision, Values, Strategies, Need Assessment and Scope of Service of the organization.

1.3 The Board of Governance should establish and approve the measures to be monitored for the Clinical Quality of all the Medical Professionals (Ongoing Professional Practice Evolution).

1.4 The Board of Governance should approve the organization's clinical governance framework.

1.5 The Board of Governance should define and approve the roles and responsibilities of governing body, management, clinicians and the workforce.

1.6 The Board of Governance should establish and approve the governance structures, including committee and reporting structures, which should be effectively monitored in order to improve the clinical performance.

1.7 The Board of Governance should ensure that the healthcare services provided meets the diverse needs of consumers and the community.

1.8 The Board of Governance should allocate enough time and attention to quality improvement, patient safety, risk management and patient experience agenda in the meeting.

1.9 Hospital leaders should submit the monthly report to Board of Governance on all reported incidents, near misses, adverse events, sentinel events.

1.10 Hospital leaders should submit the monthly report to Board of Governance on (including but not limited to) all clinical quality, patient safety, clinical risk management, patient experience, clinical quality measures of physicians, clinical quality indicators, clinical committees performance etc.

1.11 Hospital leaders should support all leaders at all levels in order to promote and drive high-quality care and develop the skills of staff in order to change and or improve the system across the organization.

1.12 Hospital leaders should measure the Safety Culture on quarterly basis by using an appropriate and scientific tool and should ensure that staff participation rate should not be less than $40 \%$. Actions should be taken based on the data collection, aggregation, analysis and action plans.

1.13 Hospital leaders should quarterly evaluate the effectiveness of systems (including but not limited topolicies, procedures, protocols, clinical care pathways, guidelines, forms and compliance with legislation, regulation and jurisdictional requirements etc.) for supporting and improving the positive organizational leadership and culture.

1.14 Hospital leaders should conduct the periodic (preferably monthly) structured Patient Safety Leadership Rounds with a multidisciplinary team including a patient and a report should be developed along with the action plan to implement and monitor.

1.15 Hospital leaders should use a communication mechanism with patients, families and carers considering the language and other barriers and the solutions to overcome such identified barriers. All communications with patients, families and carers should be documented in the patients' health records. 
1.16 Hospital leaders should ensure that the provided information to the patients, families and carers is in a way that meets their needs, and it should be easy to understand and use.

1.17 Hospital leaders should ensure that the clinical needs of patients are addressed while they are in the hospital.

1.18 Hospital leaders should ensure that the information needs for ongoing care is provided on discharge.

1.19 Hospital leaders should provide all the resources (computers, internet, journals, books etc.) and space (Lounge, Pantry and Library etc.) for all clinical staff as per their duties, roles and responsibilities.

\section{PARTNERSHIPS WITH CONSUMER :}

2.1 Hospital leaders should conduct the Patients Experience Surveys by using the scientific tools (for inpatients, out-patients and day surgery procedures) in national and English Language by using appropriate technology on monthly basis. The collected, aggregated, analyzed data should be used for process improvement and process re-design.

2.2 Hospital leaders should involve the patient's family members or carers in the care process by proving them the required skills or training to the extent they wish.

2.3 Hospital leaders should ensure that between patients and staff at all levels of the health system there is a clear, open and respectful communication exists.

2.4 Hospital leaders should develop or adopt, implement and monitor the Patients' Rights and Responsibilities or Patients Charter as per the Ministry of Health.

2.5 Hospital leaders should monitor the effectiveness of empowerment on patient's family members or carers in their care.

2.6 Hospital leaders should follow the scientific classification of the complaints and the severity assessment code matrix for the patient's complaints. Risk should be assessed for all reported patient complaints and actions should be taken as per the hospital policies and procedures.

2.7 Hospital leaders should use the patient's complaints collected, aggregated, analyzed data for system improvement or re-design.

2.8 Hospital leaders should collect, aggregate, analyze the data on Left against Medical Advice, Discharged against Medical Advice, Left Without Being Seen and Absconded Patients on monthly basis for system improvement or re-design.

2.9 Hospital leaders should Patient and Family Advisory Committee in which patients should be involved in the meetings.

2.10 Hospital leaders should involve the patients and their families or carers in staff selection, promotion, termination etc.

2.11 Hospital leaders should ensure that hospital is Patient Friendly.

2.12 Hospital leaders should ensure that all the Clinical and Patient Experience Quality Indicators are posted on the hospital website on monthly basis.

2.13 Hospital leaders should participate in community awareness on health as per the World Health Organization and Ministry of Health national and international days. 
2.14 Hospital leaders should ensure that all Medical and Nursing Staff involve the patients and their families or care takers in their care plans and decision making.

2.15 Hospital leaders should ensure that all Medical, Nursing, Paramedical and Ancillary Services Staff shared the medical records to the patients and their families or care takers.

2.16 Hospital leaders should consider sharing experiences through patient stories, information sessions, letters, pictures, patient journeys, or presentations at meetings or training sessions for hospital staff.

2.17 Hospital leaders should ensures that all the informed consent processes are as per the hospital policy which adheres to the ministry of health or relevant regulatory author requirements.

2.18 Hospital leaders should ensures that when the information for patients, carers, families and consumers about health and health services is developed internally, the organization should involve consumers in its development and review.

\section{PERSONNEL :}

3.1 Hospital leaders should allocate and manage the staff as per the Staffing Plan (which should include minimum but not limited to the Position/Title, required education, licenses, trainings, experience, knowledge, skills, attitude, full time/part time/ locum, number of staff required etc.) and scope of the service to deliver high-quality care and to meet the needs of the patients, families and carers.

3.2 Hospital leaders should ensure that the workplace is safe with great working environment.

3.3 Hospital leaders should orient the staff based on the hospital policies (General or New Hire Orientation, Departmental Orientation and General Nursing Orientation) before they will be assigned a work for nursing and paramedical staff and grant privileges for medical staff.

3.4 Hospital leaders should ensure that all staff has signed a Job Description as per their title or position.

3.5 Hospital leaders should ensure that there is policy and procedure on Credentialing and Privileging.

3.6 Hospital leaders should ensure that there is Continuous Medical Education for all Medical, Nursing and Paramedical staff as per the relevant national standards or norms.

3.7 Hospital leaders should ensure that there is staff education, training and development program for all staff.

3.8 Hospital leaders should ensure that there is there is a process for staff complaints and grievances and collected, aggregated, analyzed data should be used for system improvement.

3.9 Hospital leaders should ensure that there is process to evaluate the staff as the relevant national laws, regulations, standards or norms which include but not limited to Early Hire Evaluation, Probationary Evaluation and Annual Evaluation.

3.10 Hospital leaders should ensure that there is process for conducting the Staff Satisfaction Survey and collected, aggregated, analyzed data should be used for system improvement.

3.11 Hospital leaders should conduct the Education and Training Need Assessment on an annual basis in order to develop the annual plan of Education and Training.

\section{CLINICAL RISK MANAGEMENT :}

4.1 Hospital leaders should develop and implement the Risk Management Plan and ensure that all departments conduct a pro-active risk assessment minimum on an annual basis and based on which they should develop the mitigation plan which should be implemented and monitored. 
4.2 Hospital leaders should ensure that all untoward incidents are reported by the staff as per the hospital policy.

4.3 Hospital leaders should ensure that the Just Culture is implemented.

4.4 Hospital leaders should ensure that the Sentinel Event Policy, Root Cause Analysis, Open Disclosure, Second Victim, Apology, and Breaking Bad News are in place and are implemented.

4.5 Hospital leaders should ensure that risk is assesses for all mortalities, morbidities, litigations, complaints and irregular discharges.

4.6 Hospital leaders should assess the effectiveness of the Risk Management Plan on an annual basis.

4.7 Hospital leaders should benchmark all the monitored measures over the period of time within the department, within the organization, national level and at international level.

4.8 Hospital leaders should submit the monthly report on all identified risks along with the mitigation plan.

4.9 Hospital leaders should ensure that all the required licenses of the building, facility, staff, services etc. are valid.

\section{CLINICAL PRACTICE :}

5.1 Clinical leaders of the hospital should develop a Medical Staff Bylaws to govern the organization, functions, and responsibilities of the medical staff which should be approved by the Board of Governance.

5.2 The Board of Directors should select a Medical Director for the Clinical Services of the hospital.

5.3 The Medical Director should select the Head of the Departments for all Clinical Departments.

5.4 The Medical Director should chair the Credentialing and Privileging Committee.

5.5 Clinical heads of the department should review their departmental mortality and morbidity cases on monthly bases.

5.6 The Medical Director should chair the clinical committees of the hospital such as-Medical Review Committee, Utilization Review Committee, Blood Utilization Committee, Tissue Review Committee, Operating Room Committee, Pharmacy and Therapeutics Committee,

5.7 Clinical leaders should develop the Clinical Care Pathways, Protocols and Guidelines based on the most common discharge diagnosis and the most common procedures performed in reach department on an annual basis with most recent references (not older than five years).

5.8 Clinical leaders should provide education and training on all the approved the Clinical Care Pathways, Protocols and Guidelines to all clinical staff.

5.9 Clinical leaders should ensure that at all levels the transition of care is seamless, very safe and patient/ families or carers were involved in this process.

5.10 Clinical leaders should ensure that the data on the safety, clinical effectiveness and person-centeredness of care is collected, analyzed and shared for the purposes of both accountability and improvement.

5.11 Clinical leaders should ensure that clinical quality measures are selected and measured, monitored and benchmarked. 
5.12 Clinical leaders should ensure that there is a process to monitor the clinical performance of the physician's “Ongoing Professional Practice Evaluation.

5.13 Clinical leaders should ensure evaluate the effectiveness of all clinical practices in order to support high-quality care.

5.14 Clinical leaders should monitor the utilization of all clinical resources and services and should review as a component of quality.

5.15 Clinical leaders should develop operational policies and procedures an active engagement of clinicians.

5.16 Clinical leaders should ensure that all clinical quality data and information is available and accessible for all clinicians to support quality improvement, patient safety, risk management and patient experience.

5.17 Clinical leaders should ensure that all clinical personnel are maintaining personal professional skills, competence and performance as per the requirements of ministry of health, national standards, hospital policies and procedures and relevant regulatory bodies and should fulfill the professional regulatory requirements and codes of conduct.

\section{EDUCATION AND TRAINING :}

6.1 Clinical leaders of the hospital should provide time and resources to attend the education and training for all clinical staff on an annual basis.

6.2 Clinical leaders of the hospital should ensure that all the clinical staff maintains the required Continuous Medical Education Credits per year as per the relevant regulatory authority as per their title or position.

6.3 Clinical leaders of the hospital should ensure that all clinical staff attend courses and conferences (Continuous Professional Development), takes relevant exams, assess regularly to check competencies and should ensure that training is appropriate.

6.4 The clinical head of the department should recommend other education and training based on the annual performance appraisals and reported common incidents.

10. CLINICAL AUDIT :

7.1 Clinical leaders of the hospital should ensure that there is an audit process in place for auditing Medical Records all Mortalities and Morbidities etc.

7.2 Clinical leaders of the hospital should use the approved comprehensive Medical Records Audit Tools for in-patients and out-patients.

7.3 Clinical leaders of the hospital should audit the clinical structures, processes and outcomes on monthly basis in order to improve quality, patient safety, risk management and patient experience.

7.4 The compliance and effectiveness of the of all implemented Clinical Policies, Clinical Care Pathways, Protocols and Guidelines should be measured and monitored by an audit for all Physicians and should be linked with their Ongoing Professional Practice Evaluation.

7.5 Clinical leaders should ensure that all the Physicians have valid clinical privileges as per their scope and the compliance should be audited on a monthly basis.

\section{CLINICAL EFFECTIVENESS :}

8.1 Clinical leaders should ensure that evidence-based approach in the management of patients is implemented. 
8.2 Clinical leaders should ensure that any change in clinical practices, developing new protocols or guidelines are based on experience and evidence if current practice is shown inadequate.

8.3 Clinical leaders should ensure that only approved and published guidelines or protocols from relevant clinical organizations or bodies should be implemented.

8.4 National Service Frameworks and other national standards to ensure optimal care (when they are not superseded by more recent and more effective treatments).

\section{RESEARCH AND DEVELOPMENT :}

9.1 Clinical leaders should ensure that all research activities should be conducted to develop the body of evidence available and enhancing the level of care provided to patients in future.

9.2 Clinical leaders should always seek to change in the light of evidence-led research.

9.3 Clinical leaders should ensure that all research activities are efficiently implemented, measured and monitored for improvement.

\section{OPENNESS :}

10.1 Poor performance and poor practice can too often thrive behind closed doors. Processes which are open to public scrutiny, while respecting individual patient and practitioner confidentiality, and which can be justified openly, are an essential part of quality assurance.

10.2 Clinical leaders should ensure that open proceedings and discussion about clinical governance issues should be a feature of the framework.

10.3 Clinical leaders should ensure that it is meeting the needs of the population it serves.

10.4 Clinical leaders should conduct the health needs assessment on an annual basis.

10.5 Clinical leaders should ensure that the system of clinical governance brings together all the elements which seek to promote quality of care.

\section{INFORMATION MANAGEMENT :}

11.1 Clinical leaders should ensure that all patients' data is accurate and up-to-date and patient's confidentiality of data is respected.

11.2 Clinical leaders should ensure that full and appropriate use of the date is made to measure quality of outcomes (e.g. through audits) and to develop services tailored to local needs.

11.3 Clinical leaders should ensure that the effectiveness of the information management in patients' health records is detecting health problems, defining priorities, identifying innovative solutions and allocating resources to improve health outcomes.

\section{FURTHER RESEARCH :}

Further research is needed to understand the impact of Zuber's Person Centered Care Clinical Governance Standards for Hospitals.

\section{PRACTICE IMPLICATIONS :}

The healthcare providers should implement Zuber's Person Centered Care Clinical Governance Standards for Hospitals.

\section{CONCLUSION :}


The study concluded with "Zuber's Person Centered Care Clinical Governance Standards for Hospitals."

\section{LIMITATIONS OF THE STUDY :}

Person Centered Care and Clinical Governance are the only limitation of this study.

\section{DIRECTIONS FOR FUTURE RESEARCH :}

In the future, research should be conducted to study the overall impact of Zuber's Person Centered Care Clinical Governance Standards for Hospitals in all types of hospitals.

\section{SOURCES OF FUNDING FOR THE STUDY :}

This study was self-funded by the author.

\section{DISCLAIMER :}

This publication contains information obtained from authentic and highly regarded sources. Reasonable effort has been made to publish reliable data and information, but the author and publisher cannot assume responsibility for the validity of all materials or for the consequences of the use.

All rights reserved. No part of this publication may be reproduced, stored in a retrieval system or transmitted, in any form, or by any means, electronic, mechanical, photocopying, recording or otherwise, without permission, in writing, from the publisher or the author.

\section{REFERENCES :}

[1] Ekram S, Rahman F. (2006). The concept of patient satisfaction as it relates to contemporary health care. TAJ, 19(1), 1-12.

[2] Shaikh, Z. M. (2016). The impact of hospital accreditation on the ambulance services satisfaction. International Journal of Emerging Research in Management \& Technology (IJERMT), 5(12), 76-84.

[3] Shaikh, Z. (2017). The Impact of Hospital Accreditation on the Patient's Satisfaction of physical therapy Department Services. International Journal of Business, Management and Allied Sciences (IJBMAS), 4(4), 143-154.

[4] Shaikh, Z. M. (2017). The Impact of Hospital Accreditation on the Patients Satisfaction of Dietary Services. International Journal of Business, Management and Allied Sciences (IJBMAS), 4(4), 1-12.

[5] Shaikh, Z. M. (2017). The Impact of Hospital Accreditation on the Patients Satisfaction of Laboratory Department Services. International Journal of Business, Management and Allied Sciences (IJBMAS), 4(2), 4277-4289.

[6] Shaikh, Z. M. (2017). The Impact of Hospital Accreditation on the Patients Satisfaction of Emergency Department Services. International Journal of Business, Management and Allied Sciences (IJBMAS), 4(3), 4330-4339.

[7] Shaikh, Z. M. (2017). The Impact of Hospital Accreditation on the Patient Satisfaction of In-Patient Department Services. International Journal of Emerging Research in Management \& Technology (IJERMT), 6(8), 368-383.

[8] Shaikh, Z. M. (2017). The Impact of Hospital Accreditation on the Patients Satisfaction of Haemodialysis Department Services. International Journal of Emerging Research in Management \& Technology (IJERMT), 6(8), 384-392.

[9] Shaikh, Z. M. (2017). The Impact of Hospital Accreditation on the Patients Satisfaction of Radiology Department Services. International Journal of Business, Management and Allied Sciences (IJBMAS), $4(1), 4120-4130$.

[10] Shaikh, Z. M. (2017). The Impact of Hospital Accreditation on the Patient's Satisfaction of Pharmacy Department Services. International Journal of Business, Management and Allied Sciences, 4(4), 189199. 
[11] Shaikh, Z. M. (2017). The Impact of Hospital Accreditation on the Patients Satisfaction of Out-Patient Department Services. International Journal of Business, Management and Allied Sciences (IJBMAS), 4(3), 4384-4398

[12] Zuber Mujeeb Shaikh (2018). Impact of National Accreditation on the Patients' Experience of Ambulance Services: A Case Study. RESEARCH REVIEW International Journal of Multidisciplinary, 3(08), 177-181.

[13] Zuber Mujeeb Shaikh. (2019). The Impact of Hospital Accreditation on the Patients' Experience of Hemodialysis Department: A Case Study. International Journal of Health Sciences and Pharmacy (IJHSP), 3(1), 31-39.

[14] Zuber Mujeeb Shaikh. (2019). The Impact of Hospital Accreditation on the Patients' Experience of Emergency Department: A Case Study. RESEARCH REVIEW International Journal of Multidisciplinary, 4(2), 803-810.

[15] Shaikh, Z. M. (2017). The Impact of Hospital Accreditation on the Completeness of Personnel Files in Human Resource Department. International Journal of Business, Management and Allied Sciences (IJBMAS), 4(4), 236-244.

[16] Shaikh, Z. M. (2018). The Impact of Hospital Accreditation on the Number of Occurrence Variance Report or Incident Reports. International Journal of Business, Management and Allied Sciences (IJBMAS), 5(1), 15-19.

[17] Shaikh, Z. M. (2018). A Comparative Study on Laboratory and Blood Bank Performance by Using the Quality Indicators. International Journal of Business, Management and Allied Sciences (IJBMAS), 5(1), $1-8$.

[18] Shaikh, Z. M., Al-Omari, A., \& Ahmed, A. (2018). The impact of CBAHI accreditation on critical care unit outcome quality measures: a case study. IJHS, 8(7), 394- 407.

[19] Shaikh, Z. M., Al-Omari, A., \& Ahmed, A. (2018). The Impact of Planetree Certification on a Nationally and Internationally Accredited Healthcare Facility and its Services. RESEARCH REVIEW International Journal of Multidisciplinary, 3(8), 318-332.

[20] Safeer Ahmad, Jitendra Singh, Mohammad Arif Kamal, and Zubair Mujeeb Shaikh, (2020). PersonCentered Care Design with Reference to Healthcare Outcomes in Saudi Arabia: An Overview. American Journal of Civil Engineering and Architecture, 8(3), 91-96.

[21] Gazala Khan (2020). The Impact of Healthcare Accreditation on Patients' Satisfaction: A Literature Review. International Journal of Health Sciences and Pharmacy (IJHSP), 4(1), 49-56.

[22] Shaikh, Zuber Mujeeb. (2020). Religious and Cultural Aspects of Hand Hygiene in Healthcare Accreditations: A Review of Literature. International Journal of Health Sciences and Pharmacy (IJHSP), 4(1), 40-48.

[23] Shaikh, Zuber Mujeeb. (2020). A Case Study on Recall of used Scopes in the Endoscopy Department by using a Failure Mode \& Effect Analysis (FMEA) Proactive Risk Management. International Journal of Health Sciences and Pharmacy (IJHSP), 4(1), 13-24.

[24] Zuber Mujeeb Shaikh. (2019). The Impact of Hospital Accreditation on the Patients' Experience of Hemodialysis Department: A Case Study. International Journal of Health Sciences and Pharmacy (IJHSP), 3(1), 31-39.

[25] Zuber Mujeeb Shaikh. (2019). The Impact of Hospital Accreditation on the Patients' Experience of Emergency Department: A Case Study. RESEARCH REVIEW International Journal of Multidisciplinary, 4(2), 803-810.

[26] Shaikh, Z. M. (2016). The impact of hospital accreditation on the ambulance services satisfaction. International Journal of Emerging Research in Management \& Technology (IJERMT), 5(12), 76-84. 
[27] Shaikh, Z. (2017). The Impact of Hospital Accreditation on the Patient's Satisfaction of Physiotherapy Department Services. International Journal of Business, Management and Allied Sciences (IJBMAS), 4(4), 143-154.

[28] Shaikh, Z. M. (2017). The Impact of Hospital Accreditation on the Patients Satisfaction of Dietary Services. International Journal of Business, Management and Allied Sciences (IJBMAS), 4(4), 1-12.

[29] Shaikh, Z. M. (2017). The Impact of Hospital Accreditation on the Patients Satisfaction of Laboratory Department Services. International Journal of Business, Management and Allied Sciences (IJBMAS), 4(2), 4277-4289.

[30] Shaikh, Z. M. (2017). The Impact of Hospital Accreditation on the Patients Satisfaction of Emergency Department Services. International Journal of Business, Management and Allied Sciences (IJBMAS), 4(3), 4330-4339.

[31] Shaikh, Z. M. (2017). The Impact of Hospital Accreditation on the Patient Satisfaction of In-Patient Department Services. International Journal of Emerging Research in Management \& Technology (IJERMT), 6(8), 368-383.

[32] Shaikh, Z. M. (2017). The Impact of Hospital Accreditation on the Patients Satisfaction of Haemodialysis Department Services. International Journal of Emerging Research in Management \& Technology (IJERMT), 6(8), 384-392.

[33] Shaikh, Z. M. (2017). The Impact of Hospital Accreditation on the Patients Satisfaction of Radiology Department Services. International Journal of Business, Management and Allied Sciences (IJBMAS), 4(1), 4120-4130.

[34] Shaikh, Z. M. (2017). The Impact of Hospital Accreditation on the Patient's Satisfaction of Pharmacy Department Services. International Journal of Business, Management and Allied Sciences, 4(4), 189199.

[35] Shaikh, Z. M. (2017). The Impact of Hospital Accreditation on the Patients Satisfaction of Out-Patient Department Services. International Journal of Business, Management and Allied Sciences (IJBMAS), 4(3), 4384-4398.

[36] Zuber Mujeeb Shaikh (2018). Impact of National Accreditation on the Patients' Experience of Ambulance Services: A Case Study. RESEARCH REVIEW International Journal of Multidisciplinary, 3(08), 177-181.

[37] Zuber Mujeeb Shaikh. (2019). The Impact of Hospital Accreditation on the Patients' Experience of Hemodialysis Department: A Case Study. International Journal of Health Sciences and Pharmacy (IJHSP), 3(1), 31-39.

[38] Zuber Mujeeb Shaikh. (2019). The Impact of Hospital Accreditation on the Patients' Experience of Emergency Department: A Case Study. RESEARCH REVIEW International Journal of Multidisciplinary, 4(2), 803-810.

[39] Shaikh, Z. M. (2017). The Impact of Hospital Accreditation on the Completeness of Personnel Files in Human Resource Department. International Journal of Business, Management and Allied Sciences (IJBMAS), 4(4), 236-244.

[40] Shaikh, Z. M. (2018). The Impact of Hospital Accreditation on the Number of Occurrence Variance Report or Incident Reports. International Journal of Business, Management and Allied Sciences (IJBMAS), 5(1), 15-19.

[41] Shaikh, Z. M. (2018). A Comparative Study on Laboratory and Blood Bank Performance by Using the Quality Indicators. International Journal of Business, Management and Allied Sciences (IJBMAS), 5(1), $1-8$.

[42] Shaikh, Z. M., Al-Omari, A., \& Ahmed, A. (2018). The impact of CBAHI accreditation on critical care unit outcome quality measures: a case study. IJHS, 8(7), 394- 407. 
[43] Shaikh, Z. M., Al-Omari, A., \& Ahmed, A. (2018). The Impact of Planetree Certification on a Nationally and Internationally Accredited Healthcare Facility and its Services. RESEARCH REVIEW International Journal of Multidisciplinary, 3(8), 318-332.

[44] Shaikh, Z., Al-Towyan, S., \& Khan, G. (2016). Critical Analysis of Patient and Family Education in JCI Accreditation and CBAHI Standards for Hospitals. International Journal of Research in Business Management (IMPACT: IJRBM), 4(3), 29-38.

[45] Shaikh, Z., Al-Towyan, S., \& Khan, G. (2016). Critical Analysis of Staff Qualifications and Education Standards in JCI and Medical Staff \& Staffing Management Standards in DNV Accreditation for Hospitals. International Journal of Research in Business Management (IMPACT: IJRBM), 4(3), 61-70.

[46] Shaikh, Z., Al-Towyan, S., \& Khan, G. (2016). Critical Analysis of International Patient Safety Goals Standards in JCI Accreditation and CBAHI Standards for Hospitals. International Journal of Research in Business Management (IMPACT: IJRBM), 4(3), 71-78.

[47] Shaikh, Zuber. (2017). Critical Analysis of Patient and Family Rights in JCI Accreditation and CBAHI Standards for Hospitals. International Journal of Emerging Research in Management \& Technology (IJERMT), 6(7), 324-330.

[48] Shaikh, Zuber Mujeeb. (2020). A Case Study on Recall of used Scopes in the Endoscopy Department by using a Failure Mode \& Effect Analysis (FMEA) Proactive Risk Management. International Journal of Health Sciences and Pharmacy (IJHSP), 4(1), 13-24.

[49] Shaikh, Zuber Mujeeb. (2020). Religious and Cultural Aspects of Hand Hygiene in Healthcare Accreditations: A Review of Literature. International Journal of Health Sciences and Pharmacy (IJHSP), 4(1), 40-48.

[50] Stewart, Lee. (2007). How does a clinical governance framework contribute to the changing role of nurse leaders in Fiji, (Doctoral dissertation, James Cook University).

[51] WHO. Ninth futures forum on health systems governance and public participation. Copenhagen: WHO; 2006.

[52] WHO. (2004). Declaration of Alma-Ata international conference on primary health care, Alma-Ata, USSR, 6-12 September 1978. Development, 47(2), 159-61.

[53] Australian Commission on Safety and Quality in Health Care. Safety and quality improvement guide standard 2: partnering with consumers. Sydney: ACSQHC, 2012.

[54] Behzadifar, M., Bragazzi, N.L., Arab-Zozani, M. et al. (2019). The challenges of implementation of clinical governance in Iran: a meta-synthesis of qualitative studies. Health Res Policy Sys., 17(3), 1-14.

[55] Dwyer, Alison. (2019). Clinical Governance and Risk Management for Medical Administrators. In Textbook of Medical Administration and Leadership (pp. 99-125). Springer, Singapore.

[56] Chiozza ML, Plebani M. (2006). Clinical Governance: from clinical risk management to continuous quality improvement. Clin Chem Lab Med. 44(6), 694-698.

[57] Farokhzadian J, Dehghan Nayeri N, Borhani F. (2015). Assessment of Clinical Risk Management System in Hospitals: An Approach for Quality Improvement. Glob J Health Sci. 7(5), 294-303.

[58] Levy, B. \& Rockall, Timothy (2009). The role of clinical audit in clinical governance. Surgery (oxford). 27(1), 367-370.

[59] Chambers, Ruth \& Boath, Elizabeth \& Rogers, David. (2018). Clinical Effectiveness and Clinical Governance Made Easy. CRC Press.

[60] Newman DW, Kellett S, Beail N. (2003). From research and development to practice-based evidence: clinical governance initiatives in a service for adults with mild intellectual disability and mental health needs. J Intellect Disabil Res., 47(1), 68-74. 